\title{
A study of deployable center-fed reflectarray antenna
}

\author{
Hiromasa Nakajima ${ }^{1,}$ a), Shin-ichi Yamamoto ${ }^{1}$, \\ Michio Takikawa ${ }^{1}$, Syuji Nuimura ${ }^{1}$, and Yoshio Inasawa ${ }^{1}$ \\ ${ }^{1}$ Mitsubishi Electric Corporation, \\ 5-1-1 Ofuna, Kamakura, 247-8501, Japan \\ a)Nakajima.Hiromasa@dn.MitsubishiElectric.co.jp
}

\begin{abstract}
In this paper a deployable center-fed reflectarray antenna for mobile satellite communication systems is proposed. The proposed antenna consists of a main-substrate, four foldable sub-substrates, and a backfire antenna. This antenna can speedily be installed by attaching the backfire antenna and deploying the folded sub-substrates. The design results show that the antenna can satisfy the target gain by adjusting the deployment angle of the sub-substrates. In addition, the validity of the design is verified by measurements.
\end{abstract}

Keywords: reflectarray, deployable antenna, backfire, foldable substrate Classification: Antennas and Propagation

\section{References}

[1] J. Shinohara, N. Michishita, Y. Yamada, M.T. Islam, and N. Misran, "Design of an array feed offset parabolic reflector antenna for a simple portable earth station," 2011 International Conference on Space Science and Communication (IconSpace 2011), Penang, Malaysia, pp. 7-10, July 2011. DOI: 10.1109/iconspace. 2011.6015841

[2] M.T.H. Khan, W. Sharmin, A.K. Kundu, M.O. Goni, and K.A. Barket, "Design and performance analysis of a parabolic-inverted helix antenna and link budget optimization," 2014 17th International Conference on Computer and Information Technology (ICCIT 2014), Dhaka, Bangladesh, pp. 467-471, Dec. 2014. DOI: 10.1109/iccitechn.2014.7073078

[3] H. Nakajima, S. Yamamoto, M. Takikawa, S. Nuimura, and Y. Inasawa, "Design of deployable center-fed reflectarray antenna," International Symposium on Antennas and Propagation (ISAP2020), Osaka, Japan, pp. 625-626, Jan. 2021. DOI: 10.23919/isap47053.2021.9391436

[4] J. Shaker and M.R. Chaharmir, Reflectarray Antennas: Analysis, Design, Fabrication, and Measurement, Artech House, London, 2013.

[5] H. Legay, D. Bresciani, E. Labiole, R. Chiniard, R. Gillard, and G. Toso, "Measurement of a 1.3 m reflectarray antenna in flat panels in Ku band," 2012 International Symposium on Antennas and Propagation (ISAP), Nagoya, Japan, Oct. 2012. 


\section{Introduction}

Currently parabola antennas are often used in mobile satellite communication systems $[1,2]$. These antennas can achieve high gain easily, but they have problems in terms of portable size and installation time because the curved reflector must be divided in order to pack it in a baggage.

This paper proposes a center-fed deployable reflectarray antenna for mobile satellite communication systems [3]. Figure 1(a) shows the structure of the proposed antenna. This antenna consists of a main-substrate, four foldable sub-substrates, and a backfire antenna. Figure 1(b) shows the comparison of the antenna installation process between the conventional and proposed antennas. In order to install the conventional antenna, the antenna parts need to be unboxed, and then the supporting structures must be built. After that, the backfire antenna is attached, and the divided curved reflector is assembled. It is not so easy to install the conventional antenna. On the other hand, the proposed antenna can speedily be installed by attaching the backfire antenna and deploying the substrates. It takes less than five minutes, which is approximately a half of the conventional one. Therefore, this antenna is a good candidate for mobile satellite communication systems as it can achieve high gain using flat substrates that are easy to pack and carry. In this paper, this reflectarray antenna is designed, and its characteristics are analyzed. In addition, the validity of the design is verified by measurements.

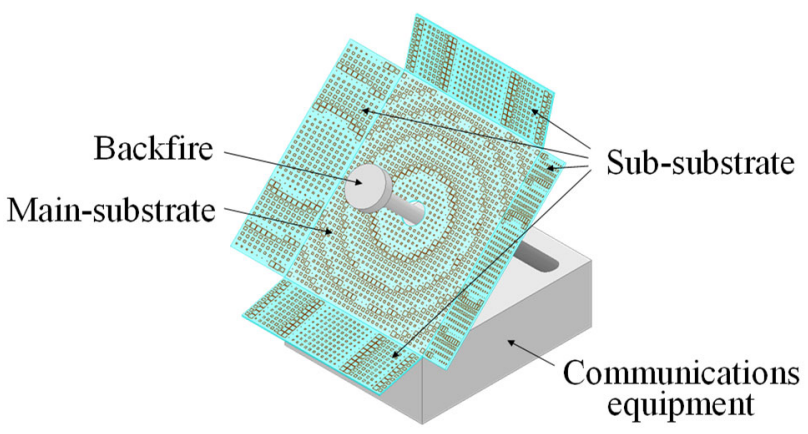

(a) Structure of the proposed antenna.

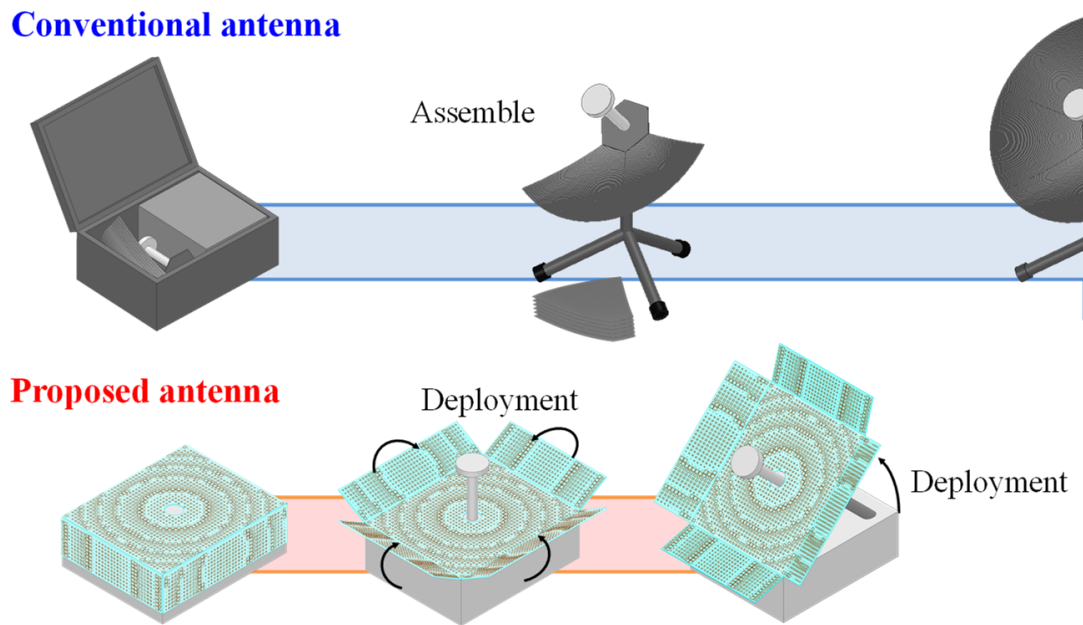

(b) Antenna installation process.

Fig. 1. Antenna structures and installation processes. 


\section{Design}

In this section, the proposed antenna is designed and analyzed.

Figures 2(a) and 2(b) show side view images of the antenna, where $\theta_{\mathrm{S}}$ shows the deployment angle of sub-substrates. In the case of $\theta_{\mathrm{s}}=0$ degrees, the path length changes depending on the reflection point. When $\theta_{\mathrm{s}}=30$ degrees, the path length difference is small. These path length differences cause a narrow bandwidth of the antenna $[4,5]$. Therefore, the $\theta_{\mathrm{s}}$ is a key parameter in our antenna design.

Figure 2(c) shows the design conditions. An existing backfire antenna is used as the primary feed. Therefore, the characteristics of the primary feed cannot be changed. $\theta_{\mathrm{s}}$ is changed from 0 to 60 degrees.

The design and analysis process of the antenna is briefly explained as follows. First, the phase distribution on the substrates from the backfire antenna is calculated. Second, the reflection phase at each element as a function of element size is calculated using a periodic boundary model. Then, each element size is determined so that the sum of the phase shift from the backfire antenna and the reflection phase of the element is uniform across the aperture surface at the design frequency. Finally, the radiation characteristics are calculated.

Figure 2(d) shows the calculated gain of the antenna for several deployment angles. As the deployment angle increases, the path length difference and the spillover loss decrease, but the antenna aperture size also decreases. As the results, $\theta_{\mathrm{s}}=30$ degrees (see Fig. 2(e)) is the optimal angle to achieve high gain over the target frequency band.

Figures 2(f) and 2(g) show the radiation patterns in the AZ plane when $\theta_{\mathrm{s}}=0$ and 30 degrees, respectively. In the case of $\theta_{\mathrm{s}}=0$ degrees, the side lobe levels are high especially at 100 degrees. It is caused by spill-over. The antenna for satellite communication systems must satisfy the side lobe regulations. In this case, it is difficult to satisfy these regulations. On the other hand, when $\theta_{\mathrm{s}}=30$ degrees, the side lobe levels are improved.

\section{Measurement}

Based on the design results in Section 2, a protype antenna is manufactured and measured to confirm the validity of the design.

Figure 3(a) shows the calculated and measured gain. The measurement results are in good agreement with the calculated results as a whole. The difference between the measured and calculated gain is less than $0.28 \mathrm{~dB}$.

Figures 3(b) and 3(c) show the radiation patterns. In the Co-pol results, the calculated and measured results are in good agreement across the wide angle. On the other hand, in the X-pol results, there is a difference in the front direction $\left(\theta_{\mathrm{s}}=0\right.$ degrees). This would be caused by the effect of POL (polarizer)/OMT (orthomode transducer) which is not considered in the design.

\section{Conclusion}

We have proposed the center-fed deployable reflectarray antenna for mobile satellite communication systems. The proposed antenna consists of a backfire antenna and foldable flat substrates. This antenna is easy to install by attaching the backfire 


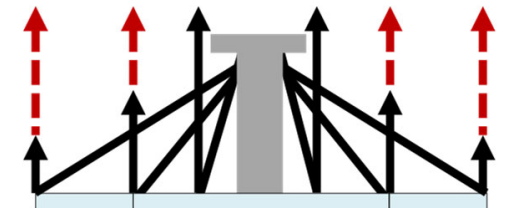

Path length difference

(a) Side view of the antenna ( $\theta_{\mathrm{s}}=0$ degrees).

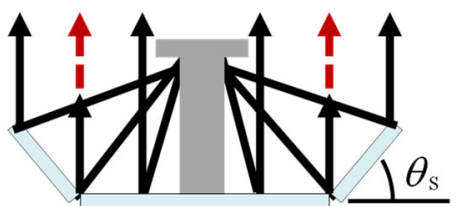

- Path length difference

(b) Side view of the antenna ( $\theta_{\mathrm{s}}=30$ degrees).

\begin{tabular}{|l|c|}
\hline Frequency band & $0.93 \mathrm{f}_{0}-1.05 \mathrm{f}_{0}$ \\
\hline Polarization & Circular \\
\hline Deployment angle $\theta_{\mathrm{s}}$ & $0-60$ degrees \\
\hline Element figure & Rectangular ring patch \\
\hline Element allocate & Rectangular \\
\hline Primary feed & Backfire \\
\hline
\end{tabular}

(c) Design conditions.

Deployment angle $\theta_{\mathrm{s}}$

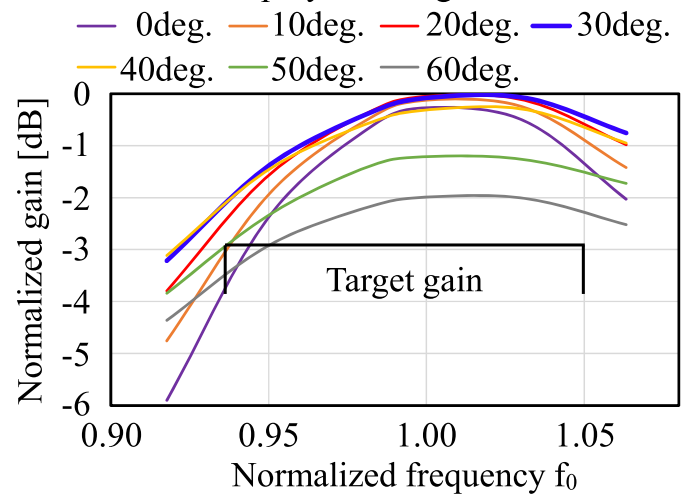

(d) Calculated gain in each deployment angle.

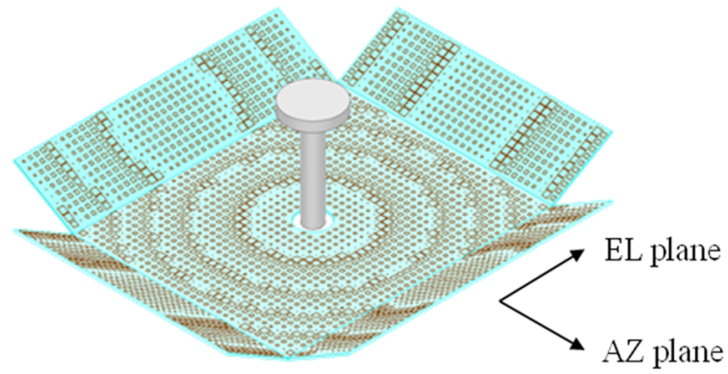

(e) Designed reflectarray antenna.

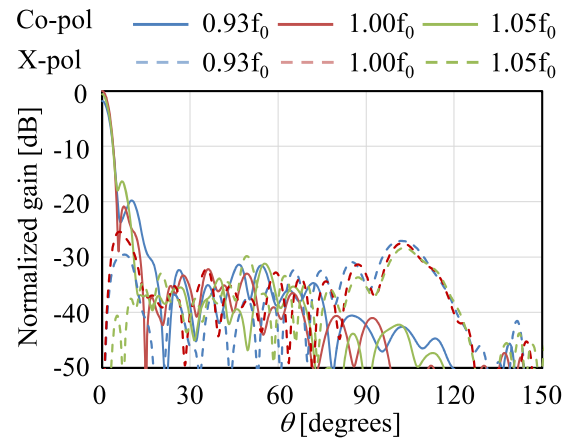

(f) Radiation pattern ( $\theta_{\mathrm{s}}=0$ degrees).

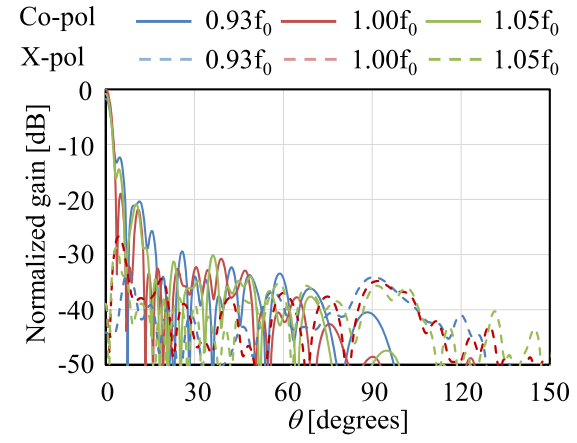

(g) Radiation pattern $\left(\theta_{\mathrm{s}}=30\right.$ degrees $)$.

Fig. 2. Configuration and design results. 


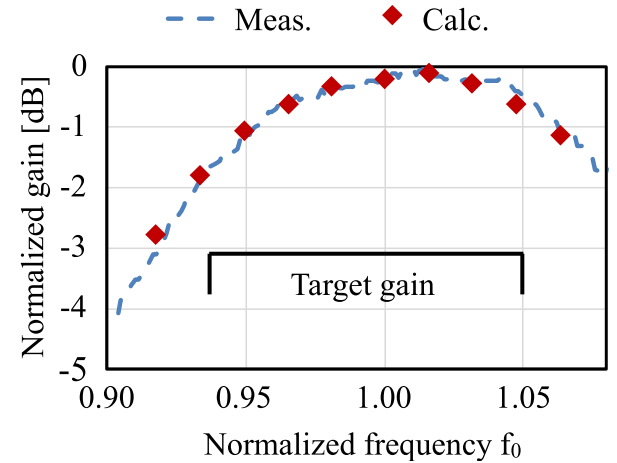

(a) Gain.

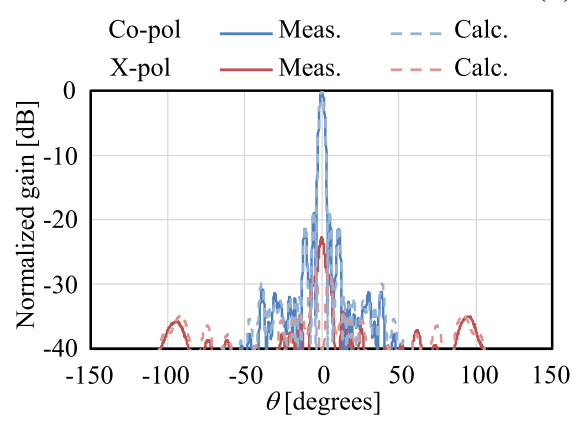

(b) Radiation patten @ $\mathrm{f}_{0}$ (Wide angle).

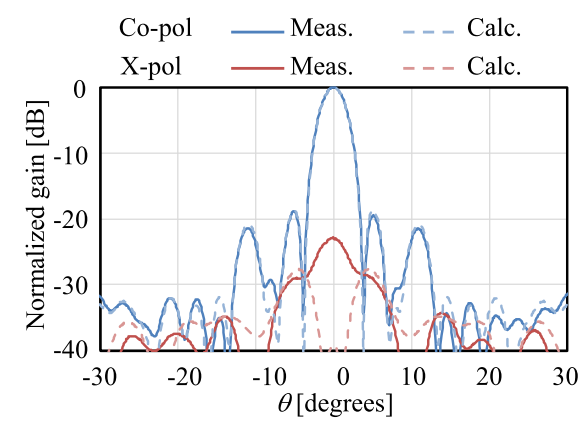

(c) Radiation patten @ $\mathrm{f}_{0}$ (Narrow angle).

Fig. 3. Measurement results.

antenna and deploying the substrates. In addition, it can be stored compactly for carrying. It has been shown that the designed antenna can satisfy the target gain by adjusting the deployment angle. In addition, the validity of the design has been verified by measurements. 